\title{
A Review of Gun Barrel Observation and Detection System

\author{
Yingxiu $\mathrm{CaO}^{\mathrm{a})}$, Liping Zheng ${ }^{\mathrm{b})}$ and Jianjie $\mathrm{Zhu}^{\mathrm{c}}{ }^{\mathrm{C}}$ \\ Mechanical Engineering College,Hebei 050003,China, \\ a)mingxiu0910@126.com, ${ }^{\text {b) } 13191885181 @ 163 . c o m,{ }^{c)} 2451396862 @ q q . c o m}$
}

Keywords: barrel defect detection; machine vision technology; detection software

\begin{abstract}
In this paper, the principle and key technology of gun barrel observation and detection are systematically expounded, and the research actuality of barrel observation and detection system is summarized and compared. This paper focuses on the application of machine vision technology in the detection of inner flaw. At last, the development of the barrel inspection and inspection system is prospected.
\end{abstract}

\section{INTRODUCTION}

Artillery is the world's army equipment, the largest number, one of the most frequently used types of weapons, it is not only the protection of military action, and will remain the ultimate occupation of the position and the main weapon of victory in modern warfare is a ground war in the possession of an irreplaceable important position ${ }^{[1]}$. The barrel is an important part of artillery, its quality directly affects the safety of gun firing, reliability, firing accuracy, artillery flight attitude and artillery life.

\section{PRINCIPLE AND KEY TECHNOLOGY OF GUN BARREL DETECTION}

\subsection{Principle of gun barrel detection}

The hardware part includes image acquisition lens, positioning and centering device, computer and accessories. The software part includes control software, image analysis software and database management software. The hardware part and the software part are composed of hardware and software. The working principle is that the CCD camera with the image acquisition lens into the bore, by hand or motor to drive the CCD camera in the bore depth and 360-degree view of the scope of detection; collected flaw images through the machine vision Technology to deal with and match the flaw database, can calculate the flaw image area and identify the flaw type ${ }^{[2]}$, draw conclusions, determine the barrel level and life, and put forward the corresponding maintenance recommendations.

\subsection{The Key Technology of Detecting Artillery Barrel}

\subsubsection{Image acquisition lens}

Gun barrel detection system generally used CCD image acquisition camera. CCD is called charge-coupled device, is a new type of semiconductor optoelectronic devices, with small size, low power consumption, large dynamic range, high sensitivity ${ }^{[3]}$, and optical systems in particular, the combination of the optical sensor will be raised to a new level .

\subsubsection{Centering positioning device}

Gun barrel detection system to ensure that each CCD camera from the subject matter of the same distance, easy to obtain the image through the image stitching technology spliced together; and to ensure that each shot the same gun when the CCD camera position the same, or two There was no significant contrast between the images acquired and the next stage of the barrel state could not be inferred. Centering positioning device CCD camera will be placed in the barrel axis, and can be located from the tail length, to determine the location of the flaw.

\subsubsection{Machine Vision Technology}

(1) Image stitching technology

Image stitching is a technique of seamlessly splicing two or more adjacent images to create a 
high-resolution image with a wide viewing angle $\mathrm{e}^{[4]}$, including image preprocessing, image registration and Image fusion, in which registration and fusion is the key technology.

\section{(2)Image matching technology}

Image matching is a certain matching algorithm in two or more images to identify the same point between the points of the process. Image matching can be divided into gray-based and feature-based matching.

\subsubsection{Software Development Technology}

Software development is based on user needs to write software development behavior, so that the system functions more practical and simple. Software is generally based on a programming language to achieve, usually using software development tools for development, in order to achieve the preparation of algorithms, software, the overall structure design and module design.

\section{RESEARCH STATUS OF GUN TUBE INSPECTION SYSTEM}

Traditionally, the artillery bore surface flaw detection by experienced technical personnel through the optical telescope system on the gun bore to observe the situation, and make the subjective judgments of the observation area, a low degree of automation, objectivity is poor, the test results Varies from person to person ${ }^{[5]}$. In recent years, with the rapid development of CCD image sensor technology and digital image processing technology, it provides the necessary technical foundation for automatic nondestructive inspection of the inner surface flaw of gun ${ }^{[6]}$.

\subsection{The development status of image acquisition lens}

Using monocular or multi-CCD camera for imaging, the greater the distortion of the image field. In order to better observe the artillery barrel bore flaw, the need for CCD camera head to optimize the design; the number of camera head design, but also the design of external mechanical structure, to achieve versatility in ground artillery, the scope is $100-150 \mathrm{~mm}$.

At present, in the gun barrel camera system applications, the vast majority of the use of unilateral CCD camera to obtain images. Single-camera installation is divided into three types, one is installed along the axis, the gun directly on the inner surface of the gun, the intake image, and then use the image processing distortion correction $^{[7]}$; Second, radial installation, the camera perpendicular to the inside Bore image, but the field of view is too small, you need to shoot several times to get the bore image ${ }^{[8]}$; Third, along the axis of the installation, the front of the camera to install the mirror, and the camera int0o a 45 degrees, by rotating the mirror to get the bore image ${ }^{[9]}$, similar to the radial installation.

\subsection{Research Status of Machine Vision Technology \\ 3.2.1 Research Status of Image Mosaic Technology}

Image stitching technology is the use of redundant information between images to shoot the image stitching together in the field of image applications widely. Using CUDA parallel computing re-compilation method and software ${ }^{[10]}$, it can overcome the limitation in traditional image mosaic, realize multi-view seamless image mosaic under the condition of scale and illumination change, and improve the speed and efficiency of image mosaic;The method of Harris-SIFT feature points extraction is proposed $^{[11]}$. The feature points are matched by the nearest neighbor method, and the feature points of the rough matching are selected by the random sampling algorithm. At the same time, Finally, the weighted average fusion algorithm is used to eliminate the stitching at the image mosaic, so as to realize the high-quality splicing of the image.

At present, there are many researches on image mosaic algorithms, which are mainly divided into two categories: region-based mosaic method and feature-based mosaic method. Image mosaic algorithm generally includes four basic steps of preprocessing, matching, establishing mathematical transformation model, image transformation and reconstruction. The matching template is established by the pixel gray value in the image or the region after the image transformation, and the corresponding position of a template or a certain point in the image to be concatenated in the reference image is found in the overlapping region of the two images; The mathematical model is established, and the mathematical model is used to transform the image to be stitched into the 
coordinate system of the reference image to obtain a smooth and seamless panorama. image.

\subsubsection{Research on Image Matching Technology}

Nowadays, a large number of image matching methods have emerged, which are aimed at improving the accuracy, speed and matching method of image matching, and the anti-jamming. A new matching algorithm based on coarse-to-fine two-step fast image matching ${ }^{[12]}$ and rough matching stage is proposed. The two-histogram (TCH) hash algorithm is used for template matching, the most similar image region is found and the ORB feature extraction And the high-speed ORB operator is used to extract and describe the feature points. Finally, the Euclidean distance method is used to match the feature vectors in the most similar region. The range of feature extraction is reduced to a small region, and the total matching time is greatly reduced. Scale invariant feature image matching algorithm ${ }^{[13]}$ uses the Gaussian pyramid model to extract the feature points in multi-scale, and then use the main direction estimation and gradient histogram method to construct the feature descriptor under the support area of the feature point. Finally, The spatial distance between the sub-pixels matches the optimal feature point. The proposed algorithm has good performance in image matching with scaled deformation, but it is not robust to illumination change.

Image matching methods have their own advantages and disadvantages. The gray-based image matching algorithm has the advantages of no additional processing, simple and easy to implement. But it is difficult to apply to image matching with scaling, rotation and partial occlusion. In contrast, feature-based image matching method can greatly reduce the impact of noise and illumination changes, good stability, strong robustness to local occlusion, and can adapt to affine transformations including rotation, scaling and other transformations. However, this kind of method has the shortcomings of relatively narrow scope of application, and the overall performance of matching depends largely on the quantity and quality of feature extraction, and this method is more suitable for the matching between images with significant features . In practical applications, the appropriate method should be chosen according to the specific situation, if a variety of methods appropriate integrated application, play their respective advantages, will improve the overall performance of image matching.

\section{RESEARCH DIRECTION OF ARTILLERY BARREL DETECTION SYSTEM}

Artillery barrel as an important part of artillery, and artillery barrel life determines the life of artillery. Various military academies and military enterprises on the body control peeping system research more generally can be divided into the following three development directions: In the acquisition lens head count, from the beginning of the monocular lens toward the binocular, multi-objective lens direction. Monocular lens field of view is small, small distortion.Binocular lens to get the flaw of the three-dimensional shape and spatial location. Multi-purpose acquisition lens sets the two advantages, both to ensure that the image of small distortion, but also access to multiple lens images, while the effect of both binocular lens. When the number of lenses increases, the front-end structure will be more complex. Therefore, looking for the number of acquisition lens and the structural complexity of the optimal point will be the focus of the study. In the optimization of image processing algorithms, has not adapted to all types of images,the future direction of development is mainly two aspects: First, the efficiency of processing algorithms to ensure image processing results while reducing processing time, can be directly on the computer screen Display the processed image, make the data more intuitive; Second, to achieve the autonomy of image processing, in the input image, according to the need to obtain the results to automatically select the standard, will effectively enhance the robustness of image processing algorithms . In the software development technology, the gun barrel detection software is still in the primary development stage, you can towards functional diversification, easy operation, simple interface and other facets development. 


\section{REFERENCES}

[1] Tan Lebin.Introduction to artillery[M]. Beijing: Beijing Institute of Technology Press ,2014:2-3.

[2] Zhang Zhenyou,Yang Qizi. Research on Digital Detector for Detecting the Flaws of Anti-aircraft Artillery Barrel[J].Acta Armamentarii, 2015,36(4):590-594.

[3] Bai Qingben. Automatic Detection of Cannon's Chamber Based on CCD Imaging and Computer Image Processing Technique[J]. Computer Measurement \& Control,2002,10(2):124-126.

[4] Zhu Chuanbei,Chen Lin. Research and Application of Image Stitching Technology[J]. Computer Knowledge and Technology, 2015,11(1): 150-156.

[5] Li Baozhang,Gu Jinjun. Exploitation of gun borehole peephole and caliper system[J]. Transd Microsys Technol,2010(6):92-94.

[6] Wang Guohui,Zheng Geyi.The Design and Realization of the Detection System for the Barrel Inside the Body [J] .Journal of Test And Measurement Technology,2010,24(5):393-396.

[7] Fu Jianping,Zhang Lihua,Lei Jie. Study of the Bore Panoramic Spying Equipment Based an Machine[J]. Journal of Gun Launch \& Control,2012(2):88-91.

[8] Yu Honglong,Wu Yongliang. Automatic Recognition Techniques of Artillery Bore Flaw Based on Jointing Algorithm[J]. Journal of Academy of Armored Force Engineering,2012,26(5):51-58.

[9] Xu Hong,Yang Ziguang. Gun Bore Damage Detection Based on The Pixel Level[J]. Computer Measurement \& Control,2013,21(9):125-128.

[10]Wang Liangliang,Zhao Shuguang. CUDA and its application in digital image mosaic[J]. Microcomputer\&ItsApplications,2013,32(2):34-40.

[11]Guo Xiaoran,Cui Shaohui.Image Mosaic Approach using Local Feature Points Registration[J]. Semiconductor Optoelectronics,2014,35(1):89-94.

[12]Qiu Lijun,Tang Jiashan. A New Fast Two-step Image Matching Algorithm[J].Computer Technology and Development,2015,25(8):67-70.

[13]G.Lowe David.Distinctive image features from scale-invariant keypoints[J].International Journal of Computer Vision,2004,60(2): 91-110. 